\title{
Waterless Hand Cleansing with Chlorhexidine during the Neonatal Period by Mothers and Other Household Members: Findings from a Randomized Controlled Trial
}

\author{
Pavani K. Ram, ${ }^{1 \star}$ Farzana Begum, ${ }^{2}$ Christina Crabtree-Ide, ${ }^{1}$ Mohammad Rofi Uddin, ${ }^{2}$ Anne M. Weaver, ${ }^{1}$ \\ Md. Golam Dostogir Harun, ${ }^{2}$ Jelena V. Allen, ${ }^{2}$ Swapna Kumar, ${ }^{2}$ Sharifa Nasreen, ${ }^{1}$ Stephen P. Luby, ${ }^{2,3}$ and Shams El Arifeen ${ }^{2}$ \\ ${ }^{1}$ State University of New York at Buffalo, Buffalo, New York; ${ }^{2}$ icddr,b, Dhaka, Bangladesh; ${ }^{3}$ Stanford University, Palo Alto, California
}

\begin{abstract}
Observational data suggest maternal handwashing with soap prevents neonatal mortality. We tested the impact of a chlorhexidine-based waterless hand cleansing promotion on the behavior of mothers and other household members. In rural Bangladesh in 2014, we randomized consenting pregnant women to chlorhexidine provision and hand cleansing promotion or standard practices. We compared hand cleansing with chlorhexidine or handwashing with soap before baby care, among mothers and household members in the two groups, and measured chlorhexidine use in the intervention arm. Chlorhexidine was observed in the baby's sleep space in $97 \%$ of 130 intervention homes, versus soap in $59 \%$ of 128 control homes. Hand cleansing before baby care was observed 5.6 times more frequently among mothers in the intervention arm than in the controls $(95 \% \mathrm{Cl}=4.0-7.7)$. Hand cleansing was significantly more frequently observed in the intervention arm among women other than the mother $(R R=10.9)$ and girls $(R R=37.0)$. Men and boys in the intervention arm cleansed hands before $29 \%$ and $44 \%$ of baby care events, respectively, compared with $0 \%$ in the control arm. The median number of grams consumed during the neonatal period was 176 (IQR =95-305 g), about $7.8 \mathrm{~g} / \mathrm{day}$ (IQR= 4.2-13.8 g). Promotion of waterless chlorhexidine increased hand cleansing behavior among mothers and other household members. Discrepancy between observed use and measured chlorhexidine consumption suggested courtesy bias in structured observations. A waterless hand cleanser may represent one component of the multimodal strategies to prevent neonatal infections in low-resource settings.
\end{abstract}

\section{INTRODUCTION}

The neonatal period (the first 28 days of life) is highly vulnerable, with $44 \%$ (2.8 million) of childhood deaths occurring in the first 28 days of life. ${ }^{1}$ Of these, nearly one-quarter are attributable to infectious causes, including sepsis, pneumonia, and umbilical cord infections. Data from observational studies suggest that hand hygiene may reduce the risk of umbilical cord infections by about $25 \%$ and mortality by $41 \%{ }^{2,3}$ But, consistent with weak habits and social norms regarding handwashing with soap in many low-income settings, mothers often do not wash their hands despite the vulnerability of the neonatal period. ${ }^{4,5}$ Hand hygiene promotion is a relatively weak component of neonatal care counseling, typically consisting of provision of a bar of soap, a basic explanation of the potential health benefits of handwashing, and instructions on how to wash hands. When applied in a cluster-randomized controlled trial in Pakistan, this approach to hand hygiene promotion did not yield benefits for neonatal mortality prevention, but because handwashing behavior was not systematically assessed, the extent to which the intervention resulted in improved handwashing is unclear. ${ }^{6}$ Indeed, prior evidence suggests that interventions relying primarily on improving awareness of the health effects of washing hands appear ineffective, likely because they do not take into account the critical psychosocial and contextual motivators of and barriers to handwashing with soap. ${ }^{5}$

In qualitative research in two sites in rural Bangladesh, we have found a number of barriers to washing hands with soap and water in the neonatal period. ${ }^{5}$ Mothers of neonates reported being too busy to wash hands. Mothers also perceived a need to avoid contact with water because of humoral

*Address correspondence to Pavani K. Ram, Department of Epidemiology and Environmental Health, State University of New York at Buffalo, 3435 Main St., Buffalo, NY 14214. E-mail: pkram@ buffalo.edu concerns, citing fears that excessive exposure to water for the mother could increase the risk of respiratory infection in the neonate. Even when mothers reported wanting to increase their handwashing behavior and to protect baby from others' germs, they found it difficult to overcome social hierarchies within their homes. Whereas mothers felt that they could ask children and, sometimes their husbands, to wash hands, they usually could not request in-laws or elders to wash hands with soap before touching the neonate.

Given the complex set of barriers to washing hands with soap and water in the newborn period, we identified the need for an innovative solution for hand cleansing that is convenient, portable, waterless, and that would be accepted by mothers and others in the home, including children and elders.

Given its bactericidal and bacteriostatic properties, chlorhexidine is used for antiseptic, topical, and dental uses worldwide; has a favorable safety profile; and is a common component of surgical scrub preparations for hand cleansing. ${ }^{7}$ Chlorhexidine is recommended for umbilical cord cleansing for neonates born at home in high neonatal mortality rate settings on the basis of multiple trials from South Asia demonstrating a reduction in all-cause neonatal mortality. ${ }^{6,8,9}$ Chlorhexidine is listed on the WHO's model list of essential medications for children for antisepsis (5\% chlorhexidine digluconate) and umbilical cord care $(7.1 \%$ chlorhexidine digluconate). A chlorhexidine-based hand cleanser has the potential to overcome numerous barriers to hand cleansing in the neonatal period because it can be delivered in a formulation that does not require rinsing and that can be maintained in a portable pump bottle at the neonate's bedside.

In this study, we sought to evaluate the behavioral effects of a chlorhexidine-based waterless hand cleansing promotion on mothers and other household members of neonates in a randomized controlled trial. Our objectives were to assess the impact of a chlorhexidine-based waterless hand cleansing promotion on the hand cleansing behavior of mothers and other household members in the neonatal period, and to 
describe the acceptability of chlorhexidine to mothers of neonates.

\section{METHODS}

Study design and site. The trial was conducted in Mirzapur, Bangladesh, a rural area approximately $60 \mathrm{~km}$ northwest of Dhaka that has one tertiary-level private hospital. Demographic surveillance in the region is ongoing and managed by Dhaka Shishu Hospital in collaboration International Centre for Diarrhoeal Diseases Research, Bangladesh.

Participant selection. The demographic surveillance system staff provided a listing of pregnant women at 3234 weeks of gestation, based on the reported date of the last menstrual period, and living in households in the demographic surveillance area. Trial data collection staff visited each woman included on the pregnancy listing to assess for the following eligibility criteria: pregnant at the time of the data collector's visit, married, between 32 and 34 weeks of gestation, and intending to reside in the demographic surveillance area through the remainder of the antenatal period and the first 4 weeks of the neonate's life. Data collectors described the study to eligible pregnant women and requested signed informed consent. Data collectors then administered a baseline survey and observed household conditions with respect to handwashing materials, water, and sanitation.

Randomization. We used block randomization, using blocks of four, to randomly assign participants to either the chlorhexidine intervention or to standard practices (control). ${ }^{10}$ The block randomization approach was used to promote balanced sample sizes across the treatment arms over the enrollment period.

An investigator with no access to study participants constructed the assignment table. A field supervisor consulted the assignment table to assign the participant to intervention or control, after baseline data collection was completed in the participant's household. Data collectors were not blinded to the assignment status of participants because the intervention included a chlorhexidine dispenser, which would have been readily observable during the data collector's visit to the household.

Description of study arms/intervention description. Intervention followed baseline data collection and assignment to the study arm. Health promoters, who were distinct from data collectors, delivered all intervention components pertinent to each treatment arm in a total of three visits to each participating household (Table 1). Health promoters provided maternal and neonatal health counseling to participants and interested family members in both arms (content shown in Box 1). Both treatment arms received a clean delivery kit, which included sufficient chlorhexidine for one-time application to the umbilical stump. No additional communication regarding hand hygiene was addressed to control participants until the completion of all data collection; in postnatal week 6 , participants in both intervention and control arms were counseled on handwashing with soap to prevent diarrhea and respiratory infections in the infant.

Hand cleansing with chlorhexidine was promoted to those in the intervention arm. The goal of the chlorhexidine hand cleansing intervention was to increase hand cleansing among mothers and others who were likely to serve as secondary caregivers, or who were likely to come into direct contact with the neonate; secondary caregivers were typically adult women, other than the mother, or older girls in the home who might provide support to the mother in caring for the newborn. The behavior change promotion focused on identifying the benefits of hand cleansing in general, the barriers faced by some to handwashing with soap, and the advantages of chlorhexidine as a waterless, portable product for hand cleansing. The discussions led by the health promoters mobilized participants to identify motivators and barriers for hand cleansing, solutions to address barriers, and strategies for reminding other household members to wash hands. In the intervention group, soap was neither encouraged nor discouraged during the course of the study.

We previously developed a theoretical model conceptualizing the individual and interpersonal determinants of hand hygiene among mothers of newborns based on the health belief model, the theory of reasoned action/theory of planned behavior, social cognitive theory, and information gleaned from formative research (Figure 1). ${ }^{11,12}$ The chlorhexidine hand cleansing intervention was delivered through an iterative discussion-oriented format that identified barriers to hand cleansing among mothers and other household members and promoted chlorhexidine for hand cleansing on the basis of three distinct benefits: nurture, convenience, and cues. Handwashing is viewed as a nurturing behavior among mothers of neonates in rural Bangladesh. ${ }^{5}$ Our intervention promoted the convenience of hand cleansing with chlorhexidine because it does not require rinsing and because it can easily be maintained at the neonate's bedside. We provided a calendar with images of the recommended times for hand

TABLE 1

Data collection and intervention schedule

\begin{tabular}{|c|c|c|c|c|c|c|c|c|}
\hline & Consent & Baseline & Randomization & Intervention & $\begin{array}{c}\text { Rapid } \\
\text { assessment }\end{array}$ & $\begin{array}{l}\text { Structured } \\
\text { observation }\end{array}$ & $\begin{array}{l}\text { Acceptability and } \\
\text { endline survey }\end{array}$ & $\begin{array}{l}\text { In-depth } \\
\text { interview }\end{array}$ \\
\hline Gestational weeks 34-36 & $\boldsymbol{\nu}^{*}$ & $\boldsymbol{\nu}$ & $\nu$ & レ & - & - & - & - \\
\hline Gestation weeks 37-39 & - & - & - & レ & - & - & - & - \\
\hline \multicolumn{9}{|l|}{ Birth } \\
\hline Postnatal week 1 & - & - & - & レレ† & $\boldsymbol{\nu}$ & - & - & - \\
\hline Postnatal week 2 & - & - & - & - & $\boldsymbol{\nu}$ & $\boldsymbol{\nu}$ & - & - \\
\hline Postnatal week 3 & - & - & - & - & - & - & - & - \\
\hline Postnatal week 4 & - & - & - & - & $\boldsymbol{\nu}$ & - & $\boldsymbol{\nu}$ & - \\
\hline Postnatal week 6 & - & - & - & $\begin{array}{l}\text { Control and intervention } \\
\text { arms (handwashing) }\end{array}$ & - & - & - & $\begin{array}{l}\text { Intervention } \\
\text { arm }\end{array}$ \\
\hline
\end{tabular}

Acted in both treatment arms.

†Two intervention visits were completed during postnatal week 1 . 


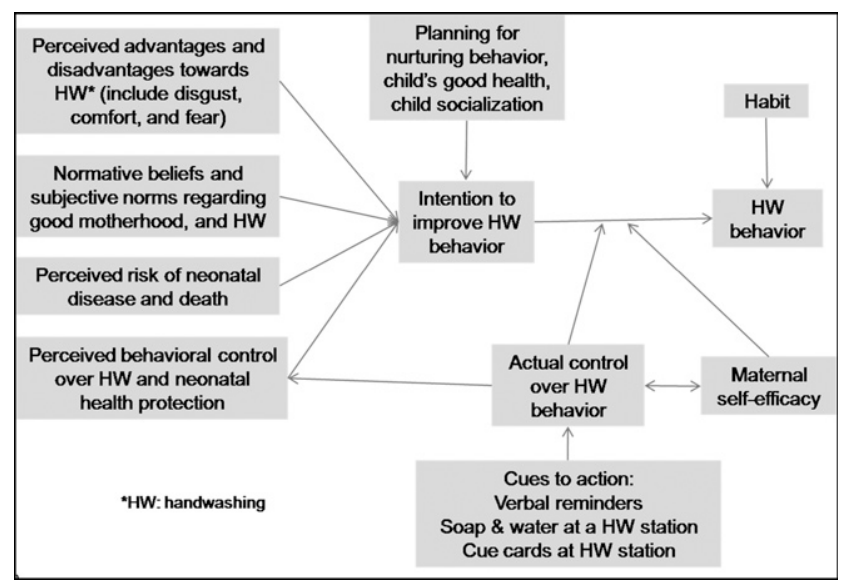

FIgURE 1. Theoretical model conceptualizing individual and interpersonal determinants of hand hygiene among mothers of newborns.

cleansing to serve as a visual cue and asked mothers to tick the dates on which they cleansed hands with chlorhexidine to promote self-reinforcement of behavior.

Among mothers and secondary caregivers (typically other adult women, adolescent girls, fathers, or others who might provide direct care for the neonate), health promoters promoted chlorhexidine hand cleansing before umbilical cord care and after contact with respiratory secretions. Among all others who might come into contact with the neonate, including children in the household and adult or child visitors from outside the household, health promoters promoted chlorhexidine hand cleansing before touching the neonate and after contact with respiratory secretions. These rolespecific times for hand cleansing will hereafter be referred to as "recommended times." Health promoters also recommended hand cleansing with chlorhexidine among mothers at three fixed times of day (morning, noon, and night) to assure at least a minimum number of hand cleanses per day.

Promoters also demonstrated chlorhexidine use to the pregnant mothers and other family members. Small children were also encouraged to participate in the demonstration events. By engaging participants in role plays with each other, health promoters encouraged mothers and family members to become comfortable with requesting each other to cleanse hands with chlorhexidine at recommended times.

Health promoters provided to each pregnant woman in the intervention group one $500-\mathrm{mL}$ bottle of $4 \%$ chlorhexidine (7.1\% chlorhexidine digluconate) lotion, ${ }^{7}$ a formulation newly developed for this study by ACI Pharmaceuticals, Ltd. (http:// www.aci-bd.com/aci-pharmaceuticals/). Participants were informed that chlorhexidine would be replenished as needed. Health promoters recommended using one pump, which, as designed, dispensed approximately $3 \mathrm{~mL}$ of the chlorhexidine product.

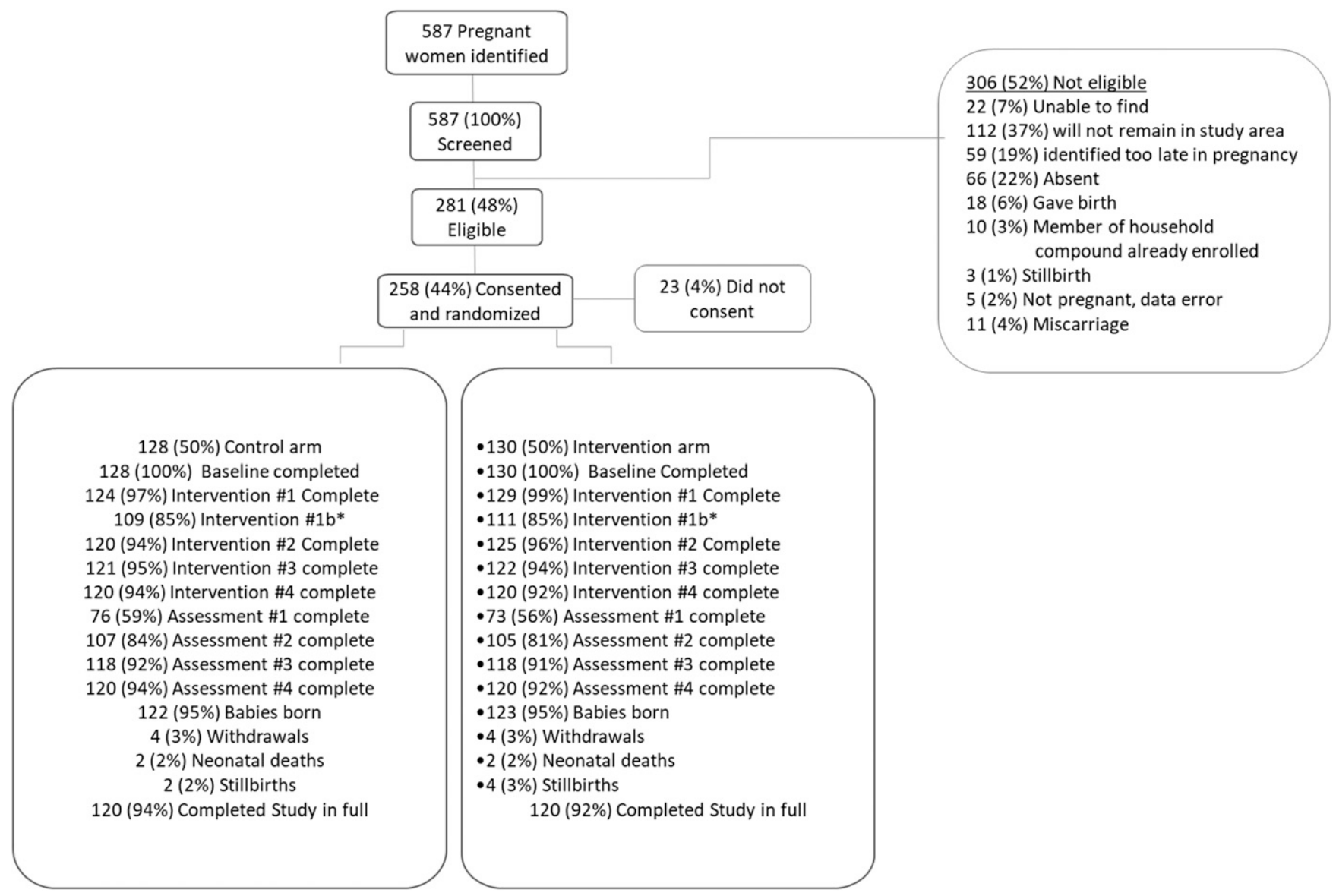

FIGURE 2. CONSORT diagram. *Intervention visit $1 \mathrm{~b}$ : because of the extensive content to be covered in the first intervention visit, some content was delivered in a follow-up interaction. 
TABLE 2

Description of participants at baseline, by treatment arm, Mirzapur, Bangladesh, 2014

\begin{tabular}{|c|c|c|}
\hline Characteristic & Control $(n=128)$ & Intervention $(n=130)$ \\
\hline Age of respondent (years), median (IQR) & $24(20-29)$ & $25(20-28)$ \\
\hline Median number of years education of respondent (IQR) & $7(5-9)$ & $7(5-9)$ \\
\hline \multicolumn{3}{|l|}{ Religion, $n(\%)$} \\
\hline Islam & $118(92)$ & $115(88)$ \\
\hline Hindu & $10(8)$ & $15(12)$ \\
\hline \multicolumn{3}{|l|}{ Household characteristics } \\
\hline Number of rooms for sleeping, median (IQR) & $2(2-3)$ & $2(1-3)$ \\
\hline Number of people living in home, median (IQR) & $4(3-6)$ & $4(3-5)$ \\
\hline Smoking inside home, $n(\%)$ & $50(39)$ & $57(44)$ \\
\hline Drinking water source: shallow tube well, $n(\%)$ & $115(90)$ & $108(83)$ \\
\hline \multicolumn{3}{|l|}{ Materials present at handwashing station, $n(\%)$} \\
\hline Water & $122(95)$ & $125(96)$ \\
\hline At least one type of soap (bar, powdered, or liquid) & $38(30)$ & $39(30)$ \\
\hline \multicolumn{3}{|c|}{ Materials reported used to wash hands after defecation, $n(\%)$} \\
\hline Water only & $76(60)$ & $79(62)$ \\
\hline Soap and water & 49 (39) & 49 (38) \\
\hline Mud & $1(1)$ & $0(0)$ \\
\hline \multicolumn{3}{|c|}{ Cleaning hands reported effective for preventing illness in the baby, $n(\%)$} \\
\hline Very effective & $124(97)$ & $122(94)$ \\
\hline Somewhat effective & $4(3)$ & $8(6)$ \\
\hline \multicolumn{3}{|l|}{ Self-report of always washing hands with soap, $n$ (\%) } \\
\hline Before eating & $120(94)$ & $122(94)$ \\
\hline After eating & $126(98)$ & $129(99)$ \\
\hline Before feeding a child & $113(88)$ & $103(79)$ \\
\hline Before touching a baby & $25(20)$ & $19(15)$ \\
\hline After cleaning a child's anus & $114(89)$ & $110(85)$ \\
\hline After disposing of a child's feces & $107(84)$ & $96(74)$ \\
\hline After defecation & $124(97)$ & 124 (95) \\
\hline After contact with respiratory secretions & $17(13)$ & $27(21)$ \\
\hline \multicolumn{3}{|l|}{ Are you able to ask others to clean their hands?, $n(\%)$} \\
\hline Always & $35(27)$ & $26(20)$ \\
\hline Sometimes & 76 (59) & 79 (61) \\
\hline Never & $17(13)$ & $25(19)$ \\
\hline
\end{tabular}

On completion of the study, intervention staff promoted handwashing with soap to participants in both intervention and control groups because of the ongoing vulnerability of the young infants to infections and the lack of availability of chlorhexidine in the market.

Birth surveillance. Field-workers encouraged birth notification by the household members and called participants once per week in weeks 37-38 and every 2 days thereafter to identify when the birth had occurred. Following birth notification, a data collector visited the home to inquire about details of the birth, use of the clean delivery kit, application of substances to the cord, and the hand hygiene of the birth attendant.

Hand cleansing measurement. Data collectors observed the presence of hand cleansing materials in the baby's sleeping room, such as soap, water, and chlorhexidine. At each of four postnatal visits, data collectors also measured the residual weight of the chlorhexidine bottle as a marker of chlorhexidine consumption.

To compare treatment arms with respect to mothers' hand cleansing behavior in the neonatal period (objective 1), our principal outcome of interest was observation of hand cleansing, which was defined as cleaning hands with chlorhexidine (intervention arm) or handwashing with soap or water (intervention or control arm). Data collectors observed hand cleansing during two 3-hour structured observations in postnatal weeks 1 and 3 . The data collector requested to observe the neonate, seated herself in as unobtrusive a position as possible, and observed all interactions that anyone had with the neonate. She observed neonatal contact and recorded whether or not hand cleansing occurred at times recommended to the intervention group.

Adverse events. Data collectors assessed potential adverse events among all participating households, recording the nature of the event and whether or not hospitalization followed the event. Skin complaints, such as rash, have been cited elsewhere as the most common adverse event resulting from chlorhexidine use. ${ }^{13}$

Acceptability. At the final visit among all intervention participants, the data collector used a structured questionnaire to inquire about the ease of chlorhexidine use, scent, skin comfort, and the occurrence of skin reactions. We conducted in-depth interviews with a subset of intervention mothers to explore barriers and motivators to the use of chlorhexidine, perceived acceptability of the chlorhexidine, and the mother's self-efficacy at asking others to use chlorhexidine before visiting with the neonate.

We conducted purposive sampling for in-depth interviews with intervention mothers according to relatively high or low use. We conducted 20 in-depth interviews including 10 mothers with high chlorhexidine use based on the weight of chlorhexidine used and/or observed use during structured observation (high use mothers) and 10 mothers who did not use chlorhexidine at all, either as observed during the structured observation or based on the weight of chlorhexidine (low use mothers). 
TABLE 3

Potential adverse events observed among mothers, fetuses, and neonates, by treatment arm, Mirzapur, Bangladesh, 2014

\begin{tabular}{|c|c|c|c|c|c|c|}
\hline & \multicolumn{3}{|c|}{ Mothers } & \multicolumn{3}{|c|}{ Fetuses/neonates } \\
\hline & Control $(n=128)(\%)$ & Intervention $(n=130)(\%)$ & $P$-value ${ }^{*}$ & Control $(n=122)(\%)$ & Intervention ( $(n=124)(\%)$ & $P$-value ${ }^{*}$ \\
\hline \multicolumn{7}{|l|}{ Mortality } \\
\hline Death & - & - & - & 2 & 2 & 1.0 \\
\hline Stillbirth & - & - & - & 2 & 4 & 0.68 \\
\hline \multicolumn{7}{|l|}{ Events with hospitalization } \\
\hline $\begin{array}{l}\text { Number of participants with serious } \\
\text { events with hospitalization }\end{array}$ & 1 & 2 & 1.0 & 7 & 13 & 0.24 \\
\hline Excessive vaginal bleeding & 1 & 1 & 1.0 & - & - & - \\
\hline Convulsion & - & - & - & 0 & 3 & 0.25 \\
\hline Diarrhea (with dehydration) & - & - & - & 0 & 1 & 1.0 \\
\hline Difficulty breathing & - & - & - & 2 & 2 & 1.0 \\
\hline Hyperthermia (fever) & - & - & - & 2 & 3 & 1.0 \\
\hline Hypothermia & - & - & - & 0 & 1 & 1.0 \\
\hline Weak, abnormal, or absent cry & - & - & - & 0 & 1 & 1.0 \\
\hline Diarrhea (no dehydration) & - & - & - & 0 & 1 & 1.0 \\
\hline Cough & - & - & - & 1 & 1 & 1.0 \\
\hline Decreased appetite & - & - & - & 0 & 1 & 1.0 \\
\hline Lethargic & - & - & - & 0 & 2 & 0.50 \\
\hline Difficulty breastfeeding & - & - & - & 0 & 1 & 1.0 \\
\hline Pus discharge from umbilicus & - & - & - & 1 & 0 & 1.0 \\
\hline Red umbilicus & - & - & - & 1 & 0 & 1.0 \\
\hline \multicolumn{7}{|l|}{ Events without hospitalization } \\
\hline $\begin{array}{l}\text { Number of participants with adverse } \\
\text { events without hospitalization }\end{array}$ & 1 & 1 & 1.0 & 19 & 11 & 0.12 \\
\hline Perineal pain & 0 & 1 & 1.0 & - & - & - \\
\hline Severe abdominal pain & 1 & 0 & 1.0 & - & - & - \\
\hline Severe headache & 1 & 0 & 1.0 & - & - & - \\
\hline Cough & - & - & - & 2 & 3 & 1.0 \\
\hline Diarrhea (no dehydration) & - & - & - & 1 & 2 & 1.0 \\
\hline Difficulty breathing & - & - & - & 3 & 0 & 0.12 \\
\hline Difficulty breastfeeding & - & - & - & 2 & 0 & 0.25 \\
\hline Hyperthermia (fever) & - & - & - & 4 & 3 & 0.72 \\
\hline Pus discharge from both eyes & - & - & - & 2 & 0 & 0.25 \\
\hline Pus discharge from umbilicus & - & - & - & 5 & 2 & 0.28 \\
\hline Skin pustules & - & - & - & 4 & 2 & 0.44 \\
\hline Ulcers or white patches in mouth & - & - & - & 1 & 3 & 0.62 \\
\hline Yellow skin or eyes & - & - & - & 1 & 1 & 1.0 \\
\hline Red umbilicus & - & - & - & 0 & 1 & 1.0 \\
\hline Swollen umbilicus & - & - & - & 2 & 1 & 1.0 \\
\hline Vomiting & - & - & - & 1 & 1 & 1.0 \\
\hline Irritable/restless & - & - & - & 1 & 0 & 1.0 \\
\hline
\end{tabular}

In addition, we conducted four focus group discussions of 22 respondents (12 females and 10 males) in total, one each among female caregivers other than the mother and male caregivers in households with high consumption of chlorhexidine and households with low consumption of chlorhexidine. We selected household members for participation in the focus group discussion by using chain referrals sampling, also referred to as snowball sampling, from among mothers with whom we conducted in-depth interviews. ${ }^{14}$

Sample size estimation. To estimate the sample size for assessing the impact of the chlorhexidine-based hand cleansing intervention on maternal handwashing behavior, we assumed that the probability of hand cleansing, that is, cleansing with chlorhexidine or handwashing with soap and water, at a recommended time would be 0.15 in the control arm, ${ }^{15}$ as measured by structured observation. We projected that the intervention would result in a $33 \%$ relative increase in observed hand cleansing by the mother at the recommended times, either by handwashing with soap or cleansing with chlorhexidine.

To observe a $33 \%$ relative increase in hand cleansing in the intervention arm, at the $95 \%$ significance level and with $80 \%$ power, we would need to observe 900 recommended times in each study arm, or 1,800 recommended times in total. To account for the effects of repeated measures from structured observation data, we applied a design effect of 2 , thus requiring us to observe 1,800 recommended times in each study arm, or 3,600 recommended times in total. Based on preliminary findings of our trial of handwashing promotion in the neonatal period in $2010-2011,{ }^{16}$ we estimated that we would observe two recommended times per hour of structured observation, indicating a need to carry out 1,800 hours of structured observation in total, or 900 hours of structured observation in each study arm. Therefore, we sought to carry out two structured observations of 3-hour duration each among 150 participants in each study arm, or 300 participants in total.

Analytic methods. We used an intent-to-treat approach for the quantitative analyses. Because the primary objective was to evaluate the effect of the intervention on the mother's complete handwashing behavior during the neonatal period, in our intent-to-treat analysis, we excluded those mothers who had fetal loss, stillbirths, or whose newborns died within the first 24 hours postpartum. 
The primary outcome of interest was the probability of hand cleansing at a recommended event by the mother of the neonate, as observed during structured observations. The secondary outcome of interest was the probability of hand cleansing at a recommended event by other household members in contact with the neonate, as observed during structured observations.

We applied the log binomial regression method to estimate the effect of intervention on the probability of hand cleansing at recommended times, using generalized estimating equations to account for clustering associated with repeated measures (i.e., numerous events, observed during two different observation periods, and for the same individual). We report risk ratios and $95 \% \mathrm{Cls}$. We first compared the intervention and control arms with respect to maternal hand cleansing behavior. Thereafter, we examined the effects of the intervention on the hand cleansing behavior of each of the following groups, accounting for clustering at the household level: adult female caregivers other than the mother ( $>12$ years old), fathers and other adult male caregivers (> 12 years old), girls, and boys.

Among participants in the intervention arm, we characterized chlorhexidine consumption based on the change in weight of chlorhexidine between visits. We described chlorhexidine consumption according to the overall weight used, the mean number of grams used per neonatal day, and the number of uses per day. Based on our observation that one pump of the chlorhexidine bottle yielded approximately $3 \mathrm{~g}$ of the product, we calculated the number of uses per day by dividing the mean number of grams used per day by 3 . Among households that received chlorhexidine replenishment during the study period, we manually calculated changes in volume to estimate the number of grams of chlorhexidine used per day. We evaluated the association between chlorhexidine consumption and the observation of maternal hand cleansing with chlorhexidine in structured observations using a generalized linear model, adjusting standard errors for clustering at the individual level.

Using survey data from among mothers in the neonatal period, we described the prevalence of self-reported acceptability of chlorhexidine for hand cleansing among respondents in the intervention arm. For qualitative data analysis, we developed a coding system based on themes commonly identified in the raw data. We examined information from participants from high-use households separately from low-use households.

This clinical trial was registered at www.clinicaltrials.gov (NCT01955317).

\section{RESULTS}

Recruitment and demographics. Substantial political unrest in 2013 delayed enrollment of participants into the study and resulted in fewer pregnant women enrolled than targeted. Among 587 women identified as pregnant from the demographic surveillance between January and July 2014, 258 (44\%) were found to be eligible and consented to participation (Figure 2). Of these, 128 (50\%) were randomly allocated to the control arm and $130(50 \%)$ to the intervention arm. Pregnancies resulted in live births in $95 \%$ of the respondents in each arm.
At baseline, respondents in the two treatment arms were similar with respect to age, educational attainment, religion, and household size (Table 2). Most households in both arms were found to have water at the main hand washing station, but only $30 \%$ in each were observed to have soap.

Adverse fetal and newborn outcomes. There were no significant differences between intervention and control groups with respect to fetal or neonatal deaths, potential adverse events with hospitalization, and potential adverse events without hospitalization (Table 3). Skin-related complaints were reported for $5 \%$ of mothers in the chlorhexidine arm, identical to the frequency in the control arm. Among neonates, skin-related complaints were reported for $41 \%$ of neonates in the control arm and $34 \%$ of neonates in the intervention arm; the difference in proportion reported in neonates across treatment arms was not statistically significant.

Availability of hand cleansing materials. During the four postnatal visits, soap was observed in the neonate's sleeping space in 59-63\% of households of control participants, compared with chlorhexidine observed in $97-99 \%$ of intervention households. Notably, soap was less commonly observed in the neonate's sleeping space in intervention households (40-61\%).

Observed hand cleansing behavior. Data collectors conducted at least one structured observation in households of 128 controls and 130 intervention participants (Table 4). In both structured observations, mothers in the control arm were more frequently observed than intervention mothers to wash hands with water alone. There was no significant difference between the two arms with respect to frequency of observed handwashing with soap. A majority of mothers in the intervention arm used chlorhexidine at least once for hand cleansing in both structured observations (week 1: 64\%; week 3: $68 \%$ ). Mothers in the intervention arm were 2.3 times as likely as controls to be observed to wash hands with soap or use chlorhexidine during the week 1 observation $(95 \% \mathrm{Cl}=$ 1.7-3.2, $P<0.0001$ ), with similar findings during the week 3 observation. Observation of hand cleansing by at least one household member other than the mother was 2.4-2.5 times as likely in intervention households compared with control households.

Data collectors observed events of recommended times for handwashing among control mothers $(n=1,441)$ and intervention mothers $(n=1,471)$ (Table 5). Hand cleansing was observed in $5 \%$ of 849 baby care events observed in control mothers and $26 \%$ of 851 events observed in intervention mothers $(\mathrm{RR}=5.6 ; 95 \% \mathrm{Cl}=3.8-8.2)$. Intervention mothers were also significantly more likely to be observed washing hands with soap or using chlorhexidine after potential contact with feces from the mother herself, newborn, or any other child $(\mathrm{RR}=4.1 ; 95 \% \mathrm{Cl}=2.9-5.8)$. Whereas none of the control mothers washed hands with soap after contact with her own or any child's respiratory secretion, hand cleansing was observed among $29 \%$ of intervention mothers at such events.

Group differences in hand cleansing were greater among household members than among mothers (Table 6). In intervention households, women other than the mother were 10.9 times more likely to be observed practicing hand cleansing before baby care than their counterparts in the control arm $(95 \% \mathrm{Cl}=4.9-24.1)$. Whereas $40 \%$ of girls cleansed hands with chlorhexidine or soap in the intervention arm, only $1 \%$ of girls in the control arm did so $(R R=37.0 ; 95 \%$ 


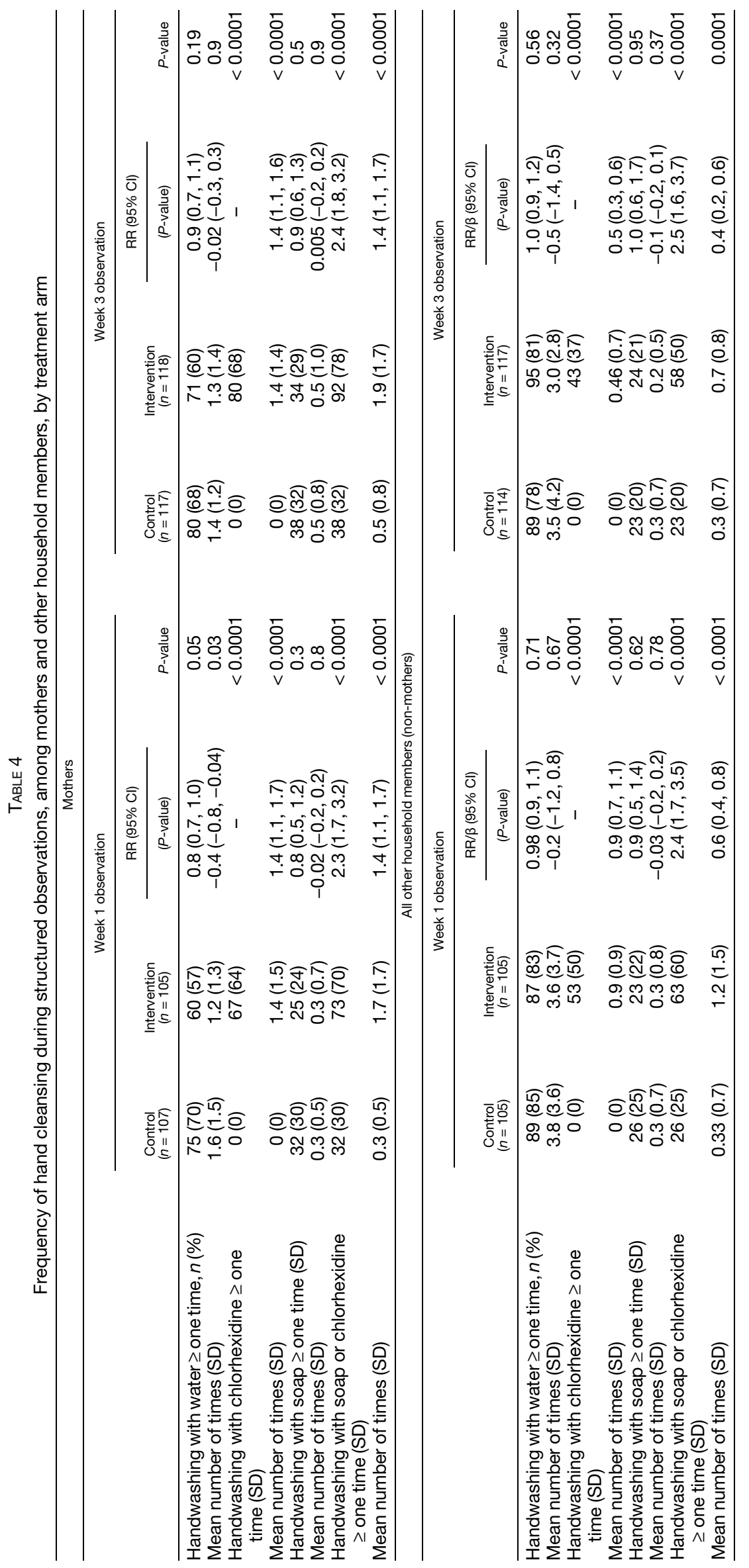


TABLE 5

Observed hand cleansing among mothers, by treatment arm, Mirzapur, Bangladesh, 2014

\begin{tabular}{|c|c|c|c|}
\hline Event type & Control & Intervention & RR $(95 \% \mathrm{Cl})$ \\
\hline \multicolumn{4}{|l|}{ Baby care } \\
\hline Number of events & 849 & 851 & - \\
\hline Proportion with hand cleansing (\%) & 5 & 26 & $5.6(4.0-7.7)$ \\
\hline \multicolumn{4}{|l|}{ Fecal contact } \\
\hline Number of events & 261 & 284 & - \\
\hline Proportion with hand cleansing (\%) & 12 & 49 & $4.1(2.9-5.9)$ \\
\hline \multicolumn{4}{|l|}{ Respiratory secretion contact } \\
\hline Number of events & 5 & 13 & - \\
\hline Proportion with hand cleansing (\%) & 0 & 29 & - \\
\hline \multicolumn{4}{|l|}{ Food contact } \\
\hline Number of events & 321 & 323 & - \\
\hline Proportion with hand cleansing (\%) & 6 & 8 & $1.4(0.8-2.6)$ \\
\hline \multicolumn{4}{|l|}{ All events } \\
\hline Number of events & 1,441 & 1,475 & - \\
\hline Proportion with hand cleansing (\%) & 6 & 26 & $4.30(3.45,5.35)$ \\
\hline
\end{tabular}

$\mathrm{Cl}=5.1-269.7)$. None of the men or boys in the control arm cleansed hands before baby care events; $29 \%$ of men and $44 \%$ of boys in the intervention arm did so.

Chlorhexidine consumption. Data collectors weighed chlorhexidine bottles at two or more visits to 120 (92\%) intervention mothers, 11 (9\%) of whom had received chlorhexidine replenishment. Among 120 with chlorhexidine weight data, the median number of grams consumed during the neonatal period was 176 (IQR $=95-305 \mathrm{~g})$, representing a median of $7.8 \mathrm{~g}$ of chlorhexidine consumed per day (IQR = 4.2-13.8 g). Hence, the estimated number of uses was a median of 2.4 uses (IQR $=1.4-4.6$ uses). Chlorhexidine consumption, as measured by weight change, was modestly correlated with the number of times that mothers cleansed hands with chlorhexidine during the structured observation $(R=0.17, P=0.01)$. Chlorhexidine use was significantly more likely to be observed in structured observations in households in the second, third, and fourth highest quartiles of chlorhexidine consumption than in households in the lowest quartile of consumption (Table 7).

Because there is a substantial risk of reactivity among subjects in structured observation, we also evaluated the approximate number of times of hand cleansing with chlorhexidine per day based on observed consumption. ${ }^{17}$ If observed use during the 3-hour structured observation (mean 1.4 times per mother) were representative of approximately 18 waking hours per day, then the daily chlorhexidine consumption would have been approximately $25 \mathrm{~g} /$ day (or approximately eight uses). Our analysis of chlorhexidine weight changes indicated a lower median consumption of $7.8 \mathrm{~g} /$ day (or $2.4 \mathrm{~g}$ uses per day) than that based on structured observations. Based on a mean 0.3 hand washes with soap during the 3-hour structured observation in the control group, we estimate that the number of times control mothers washed their hands with soap in an 18-hour day is 1.8, marginally lower than the 2.4 chlorhexidine uses in the intervention group.

Acceptability of chlorhexidine. In in-depth interviews and group discussions, participants reported that the chlorhexidine works quickly, is easy and convenient to use, and time saving; they appreciated its waterless and antimicrobial properties. Mothers reported that it was easier to ask others to clean hands with chlorhexidine than to wash hands with soap. Barriers to chlorhexidine use included a perceived long drying time, which was reported by a few mothers. Some did not like the feel of the chlorhexidine lotion. In addition, mothers did not like to use chlorhexidine before breastfeeding for several reasons, including a concern about the neonate swallowing chlorhexidine if the mother cleansed her hands before feeding.

TABLE 6

Observed hand cleansing among household members of neonates, by treatment arm, Mirzapur, Bangladesh, 2014

\begin{tabular}{|c|c|c|c|}
\hline Category of household contacts of neonates & Control & Intervention & $\mathrm{RR}(95 \% \mathrm{Cl})$ \\
\hline \multicolumn{4}{|l|}{ Mothers } \\
\hline Number of events & 849 & 851 & - \\
\hline Proportion with hand cleansing (\%) & 5 & 26 & $5.6(4.0-7.7)$ \\
\hline \multicolumn{4}{|l|}{ Fathers } \\
\hline Number of events & 1 & 3 & - \\
\hline Proportion with hand cleansing (\%) & 0 & 66 & - \\
\hline \multicolumn{4}{|l|}{ Adult women, other than the mother } \\
\hline Number of events & 224 & 215 & - \\
\hline Proportion with hand cleansing (\%) & 3 & 34 & $10.87(4.91-24.05)$ \\
\hline \multicolumn{4}{|l|}{ Adult men, other than the father } \\
\hline Number of events & 10 & 14 & - \\
\hline Proportion with hand cleansing (\%) & 0 & 29 & - \\
\hline \multicolumn{4}{|l|}{ Girls } \\
\hline Number of events & 92 & 97 & - \\
\hline Proportion with hand cleansing (\%) & 1 & 40 & $36.99(5.07-269.69)$ \\
\hline \multicolumn{4}{|l|}{ Boys } \\
\hline Number of events & 40 & 39 & - \\
\hline Proportion with hand cleansing (\%) & 0 & 44 & - \\
\hline
\end{tabular}


TABLE 7

Association between chlorhexidine consumption, as determined by change in weight, and observed handwashing with chlorhexidine among participants in the intervention arm, Mirzapur, Bangladesh, 2015

\begin{tabular}{cc}
\hline & $\begin{array}{c}\text { Association between quartile of } \\
\text { Quartile of chlorhexidine } \\
\text { consumption per day }\end{array}$ \\
\cline { 2 - 2 } & $\begin{array}{c}\text { hand cline consumption and observed } \\
\text { hansing with chlorhexidine }\end{array}$ \\
\hline & All household $\mathrm{Cl})$ \\
2 & Referent \\
3 & $1.4(1.06,2.1)$ \\
4 & $1.5(1.02,2.3)$ \\
1 & $1.8(1.2,2.8)$ \\
2 & Mothers of neonates \\
3 & Referent \\
4 & $1.8(1.13,2.8)$ \\
& $1.8(1.14,2.74)$ \\
\end{tabular}

Male participants felt that the intervention did not adequately involve them, perceiving that the health promoters more readily included other women in the family than themselves. We did not find substantive differences in reported barriers and motivators for chlorhexidine use, among participants in high-use households versus those in low-use households.

\section{DISCUSSION}

During the vulnerable and busy neonatal period, a number of barriers prevent hand hygiene among mothers and other household members of neonates. Provision and promotion of a waterless chlorhexidine-based hand cleansing option led to increases in observed hand cleansing among mothers and other household members, compared with standard practices. However, the estimated number of uses of chlorhexidine per day lagged behind the number of times recommended.
These findings underscore the continued challenge in promoting hand hygiene in the neonatal period. Extensive prior research in Bangladesh has documented the lack of a widespread handwashing habit and the dearth of social norms favoring at times of possible pathogen transmission, and mothers have described the challenges they face in improving their own handwashing or that of others in the household during the newborn period. ${ }^{5}$ The improvements we have observed in hand cleansing with provision and promotion of chlorhexidine were likely modest, given the relatively short intervention duration against the backdrop of lifelong habits, and the busy and complex newborn period. The safety profile of the chlorhexidine lotion for hand cleansing was favorable, consistent with that in other studies. ${ }^{9,18-21}$

Although handwashing with soap before umbilical cord contact has been observed to be protective against umbilical cord infections, ${ }^{22}$ there is a paucity of information on the effectiveness of hand cleansing at other times of potential pathogen transmission to neonates in low- and middle-income countries. We recommended fixed times for handwashing, at three times in the day, and event-specific times for chlorhexidine use, such as before umbilical contact, ${ }^{22}$ to reduce the risk of pathogen transmission from mothers and other family members. Adherence to such ambitious recommendations, especially the large number of times to cleanse hands, may be particularly challenging during the busy neonatal period.

Mothers and newborns are closely cocooned with each other, particularly in South Asian cultural context. ${ }^{5}$ Other household members, particularly children, may be responsible for introducing new organisms to the newborn because they are more likely to move in and out of the household environment. Compared with control peers, increases in observed hand cleansing among secondary caregivers and other household members were greater than among mothers in the intervention group. Whereas our prior work suggested that it

Box 1

Maternal and neonatal health counseling

The objectives of the maternal and neonatal health counseling were to do the following:

- Highlight the vulnerability of the neonatal period.

- Inform participant and her family of the schedule recommended by the government of Bangladesh for prenatal care and strongly encourage participants to seek appropriate prenatal care, including tetanus toxoid vaccination. Tetanus toxoid is provided free of cost as part of antenatal care at all government of Bangladesh health facilities.

- Discuss birth plans and encourage participants to develop a birth plan, including location of the birth, materials, persons needed, and an emergency plan.

- Introduce and discuss the use of a clean delivery kit for hygienic delivery to reduce infections. This will include instructions on cutting the umbilical cord. Provide a clean delivery kit to the participant, which will include a clean blade, a clean string for tying the cord, and a clean mat for the mother to lie on, among other items.

- Encourage close family members to motivate or demand hand washing with soap by birth attendants before and during labor and delivery.

- Discuss maternal danger signs with participant and her family and encourage care seeking when recognized.

- Discuss neonatal danger signs with participant and her family and encourage care seeking when recognized.

- Discuss neonatal hypothermia prevention with participant and her family and work with the family to build acceptance of the message.

- Specifically, recommend immediate drying of the newborn and placement on the mother's belly or chest and then covering.

- Delay bathing until day 2 of life.

- Discuss the benefit of immediate and exclusive breastfeeding with participant and her family and work with the family to build acceptance of the message.

- Provide chlorhexidine and gauze for umbilical cord cleansing, and explain chlorhexidine cord care to the participant and work with her and her family.

Materials: Each participant was given a clean delivery kit and a culturally appropriate pictorial card depicting each of the danger signs for the mother pre-, during, and post-labor, and for the neonate. 
was very difficult to motivate other household members to wash hands with soap, mothers in this study indicated that they were able to request others to use chlorhexidine, perhaps because it was perceived as a medical product to protect the vulnerable neonate.

Chlorhexidine's bacteriostatic properties may delay the recontamination of hands or reduce the intensity of hand contamination, giving it potential advantage over hand cleansing with soap or alcohol-based hand sanitizer. ${ }^{7}$ However, we are not aware of data from heavily contaminated lowincome community settings that characterize these potential benefits of chlorhexidine hand cleansing fully. Thus, we cannot know whether chlorhexidine represents a more efficacious product for prevention of neonatal infections than either soap or alcohol-based hand sanitizer. Likewise, we do not yet understand the potential adverse ramifications of frequent topical application of such a bacteriostatic product for the establishment of healthy newborn microbiota. ${ }^{23}$

The waterless properties afforded by chlorhexidine can also be provided by alcohol-based hand sanitizers. Chlorhexidine hand cleansing preparations are largely relegated to use in healthcare settings, whereas alcohol-based hand sanitizers are increasingly commercially available in many low- and middleincome countries. Although currently beyond the economic reach of most mothers of neonates in low-income settings such as rural Bangladesh, the pathway to scale may be more achievable for commercially available alcohol-based sanitizers than chlorhexidine hand cleansing preparations. Still, based on our analysis of chlorhexidine consumption, strategies to promote adoption and use of alcohol-based sanitizers will suffer the same challenges that have been characterized for promotion of handwashing with soap ${ }^{16}$ or, in this article, hand cleansing with chlorhexidine. Moreover, the protective effects of chlorhexidine, alcohol-based sanitizers, and soap are not entirely interchangeable. Alcohol and chlorhexidine may be poorly effective at reducing hand contamination with some pathogens relevant to the LMIC context, including nonenveloped viruses. ${ }^{24}$ Handwashing with soap and water is recommended over cleansing with alcohol when hands are grossly soiled, which may occur with some frequency among caregivers of newborns, who produce unformed stools. ${ }^{7}$

There are numerous well-documented challenges to measuring hand hygiene, and none of the widely applied methods are free of validity concerns. ${ }^{17,25}$ Structured observation, which is certainly subject to courtesy bias, yields data rich with detail regarding the timing and nature of hand cleansing behavior. In this study, structured observation allowed for comparisons between groups using very different materials for hand cleansing (chlorhexidine lotion versus bar soap). By using a secondary method of behavior measurement, chlorhexidine consumption as indicated by weight change, we were able to apply a reality filter to otherwise highly optimistic findings of behavior change. A second limitation of the study was that data collectors were not blinded to treatment arm allocation; given the hand cleansing behavioral outcome of this trial, there was no feasible way to introduce a placebo in the intervention arm.

\section{CONCLUSION}

Our data suggest the potential for a waterless hand cleanser, chlorhexidine in this study, to facilitate hand cleansing among mothers and family members of newborns. However, it is not clear whether the frequency of hand cleansing was increased sufficiently to prevent neonatal infections. Assessing health outcomes of hand cleansing promotion necessitates using a robust and costly experimental design. Public health research dollars may be better invested in developing and evaluating multimodal strategies to prevent neonatal infections in low-resource settings, including promotion of hand hygiene and umbilical cord care among families of newborns, and strengthened infection prevention and control practices in health facilities where babies are increasingly delivered.

Received October 17, 2019. Accepted for publication May 29, 2020.

Published online September 21, 2020.

Acknowledgments: We are thankful for the time and information provided by our study participants and their families, even during the busy newborn period. Our work would not have been feasible without the diligence and discipline of the entire study team of data collectors, behavior change communicators, and the field supervisors. ACI Pharmaceuticals, Ltd. graciously rose to the challenge to provide the chlorhexidine product for hand cleansing.

Financial support: This project was made possible through the generous support of the Saving Lives at Birth partners: the U.S. Agency for International Development (USAID), the government of Norway, the Bill \& Melinda Gates Foundation, Grand Challenges Canada, and the United Kingdom government. It was executed, and this article was prepared, by the named authors, and does not necessarily reflect the views of the Saving Lives at Birth partners.

Authors' addresses: Pavani K. Ram, Christina Crabtree-Ide, Anne M. Weaver, Jelena V. Allen, and Swapna Kumar, Department of Epidemiology and Environmental Health, State University of New York at Buffalo, Buffalo, NY, E-mails: pkram@buffalo.edu, crc8@buffalo.edu, aweaver@buffalo.edu, jelenavujcic@gmail.com, and swapnak28@ gmail.com. Farzana Begum, Mohammad Rofi Uddin, Md. Golam Dostogir Harun, and Sharifa Nasreen, Centre for Communicable Diseases, icddr,b, Dhaka, Bangladesh, E-mails: farzanab@wsup.com, rofi.uddin@icddrb.org, dostogirharun@icddrb.org, and drsharifa74@ gmail.com. Stephen P. Luby, Department of Medicine, Stanford University, Stanford, CA, E-mail: sluby@stanford.edu. Shams El Arifeen, Maternal and Child Health, icddr,b, Dhaka, Bangladesh, E-mail: shams@icddrb.org.

\section{REFERENCES}

1. Liu L, Oza S, Hogan D, Perin J, Rudan I, Lawn JE, Cousens S, Mathers C, Black RE, 2015. Global, regional, and national causes of child mortality in 2000-2013, with projections to inform post-2015 priorities: an updated systematic analysis. Lancet 385: 430-440.

2. Mullany LC et al., 2009. Incidence and risk factors for newborn umbilical cord infections on Pemba Island, Zanzibar, Tanzania. Pediatr Infect Dis J 28: 503-509.

3. Rhee V, Mullany LC, Khatry SK, Katz J, LeClerq SC, Darmstadt GL, Tielsch JM, 2008. Maternal and birth attendant hand washing and neonatal mortality in southern Nepal. Arch Pediatr Adolesc Med 162: 603-608.

4. Ram PK, Kumar S, 2014. Handwashing in the Perinatal Period: Literature Review and Synthesis of Qualitative Research Studies from Bangladesh, Indonesia, and Kenya. Washington, DC: MCHIP: Maternal and Child Health Integrated Program.

5. Parveen S et al., 2018. Barriers to and motivators of handwashing behavior among mothers of neonates in rural Bangladesh. BMC Public Health 18: 483.

6. Soofi S, Cousens S, Imdad A, Bhutto N, Ali N, Bhutta ZA, 2012. Topical application of chlorhexidine to neonatal umbilical cords for prevention of omphalitis and neonatal mortality in a rural district of Pakistan: a community-based, cluster-randomised trial. Lancet 379: 1029-1036.

7. CDC, 2002. Guideline for hand hygiene in health-care settings: Recommendations of the Healthcare Infection Control 
Practices Advisory Committee and the HICPAC/SHEA/APIC/ IDSA Hand Hygiene Task Force. MMWR 51: 1-56.

8. Mullany LC, Saha SK, Shah R, Islam MS, Rahman M, Islam M, Talukder RR, El Arifeen S, Darmstadt GL, Baqui AH, 2012. Impact of $4.0 \%$ chlorhexidine cord cleansing on the bacteriologic profile of the newborn umbilical stump in rural Sylhet District, Bangladesh: a community-based, cluster-randomized trial. Pediatr Infect Dis J 31: 444-450.

9. Arifeen SE et al., 2012. The effect of cord cleansing with chlorhexidine on neonatal mortality in rural Bangladesh: a community-based, cluster-randomised trial. Lancet 379: 1022-1028.

10. Altman DG, Bland JM, 1999. How to randomise. Br Med J 319: 703-704.

11. Glanz K, Rimer BK, Vishwanath K, 2002. Health Behavior and Health Education. San Francisco, CA: Jossey-Bass.

12. Pajares F, 2002. Overview of Social Cognitive Theory and of SelfEfficacy. Available at: https://www.uky.edu/ eushe2/Pajares/ eff.html. Accessed July 17, 2020.

13. Silvestri DL, McEnery-Stonelake M, 2013. Chlorhexidine: uses and adverse reactions. Dermatitis 24: 112-118.

14. Sadler GR, Lee H-C, Lim RSH, Fullerton J, 2010. Recruitment of hard-to-reach population subgroups via adaptations of the snowball sampling strategy. Nurs Health Sci 12: 369-374.

15. Curtis VA, Danquah LO, Aunger RV, 2009. Planned, motivated and habitual hygiene behaviour: an eleven country review. Health Educ Res 65: 655-673.

16. Ram PK, Nasreen S, Kamm K, Allen J, Kumar S, Rahman MA, Zaman $\mathrm{K}$, El Arifeen S, Luby SP, 2017. Impact of an intensive perinatal handwashing promotion intervention on maternal handwashing behavior in the neonatal period: findings from a randomized controlled trial in rural Bangladesh. Biomed Res Int 2017: 6081470.

17. Ram PK et al., 2010. Is structured observation a valid technique to measure handwashing behavior? Use of acceleration sensors embedded in soap to assess reactivity to structured observation. Am J Trop Med Hyg 83: 1070-1076.

18. Alam MA et al., 2008. Newborn umbilical cord and skin care in Sylhet District, Bangladesh: implications for the promotion of umbilical cord cleansing with topical chlorhexidine. J Perinatol 28 (Supp/ 2): S61-S68.

19. McClure EM et al., 2007. The use of chlorhexidine to reduce maternal and neonatal mortality and morbidity in low-resource settings. Int J Gynaecol Obstet 97: 89-94.

20. Mullany LC, Biggar RJ, 2009. Vaginal and neonatal skin cleansing with chlorhexidine. Lancet 374: 1873-1875.

21. Mullany LC, Khatry SK, Sherchand JB, LeClerq SC, Darmstadt GL, Katz J, Gauchan P, Adhikari RK, Rana A, Tielsch JM, 2008. A randomized controlled trial of the impact of chlorhexidine skin cleansing on bacterial colonization of hospital-born infants in Nepal. Pediatr Infect Dis J 27: 505-511.

22. Mullany LC, Darmstadt GL, Katz J, Khatry SK, LeClerq SC, Adhikari RK, Tielsch JM, 2007. Risk factors for umbilical cord infection among newborns of southern Nepal. Am J Epidemiol 165: 203-211.

23. Kilian M, Chapple ILC, Hannig M, Marsh PD, Meuric V, Pedersen AML, Tonetti MS, Wade WG, Zaura E, 2016. The oral microbiome - an update for oral healthcare professionals. Br Dent $J$ 221: 657-666.

24. Park GW, Barclay L, Macinga D, Charbonneau D, Pettigrew CA, Vinjé J, 2010. Comparative efficacy of seven hand sanitizers against murine norovirus, feline calicivirus, and GIl. 4 norovirus. J Food Prot 73: 2232-2238.

25. Ram P, 2013. Practical Guidance for Measuring Handwashing Behavior: 2013 Update: Water and Sanitation Program. Washington, DC: World Bank Publications. 